\title{
Low-complexity soft-decision aided detectors for coded spatial modulation MIMO systems
}

\author{
Cong Li, Jinlong Wang, Yunpeng Cheng ${ }^{*}$ and Yuzhen Huang
}

\begin{abstract}
In this paper, we present an efficient transmission scheme for multiple-input multiple-output (MIMO) systems, i.e., coded spatial modulation (SM) systems with soft-decision aided detector. To exploit the powerful error correction of channel coding, the key challenge of coded SM systems is on designing a reliable but low-complexity soft-output detector. Fighting against this problem, we first propose two soft-output detection algorithms by exploiting the features of M-phase-shift keying (PSK) and M-quadrature amplitude modulation (QAM) constellations, namely, PSK-based soft-output detector (PBSD) and QAM-based soft-output detector (QBSD). Furthermore, to further enhance the performance of the two algorithms, we propose another two soft-output detection algorithms taking into account of counterpart maximum-likelihood (ML) estimate, namely, improved PSK-based soft-output detector (IPBSD) and improved QAM-based soft-output detector (IQBSD). The findings of this paper demonstrate that: (1) The computational complexity of PBSD and QBSD algorithms are much lower than that of Max-Log-LLR algorithm at the expense of error performance. (2) Both the IPBSD and IQBSD algorithms achieve the same performance as Max-Log-LLR algorithm with reduced computational complexity. In addition, a comprehensive performance and computational complexity comparison between the proposed algorithms and the Max-Log-LLR algorithm is provided to verify our proposed low-complexity soft-output detectors.
\end{abstract}

Keywords: Spatial modulation, Multiple-input multiple-output, Maximum a posteriori probability, Max-Log-LLR detector

\section{Introduction}

Spatial modulation (SM) has been identified as a new efficient multiple-input multiple-output (MIMO) technique, which was first proposed in [1]. The main principle of $\mathrm{SM}$ is that it jointly uses antenna indices and a conventional signal set to convey information and activates only one antenna to transmit the traditional modulated symbol in each time slot. The advantages of SM, such as the relaxation of antenna synchronization, the avoidance of inter-channel interference, and the reduction in transreceiver complexity, make it become a topic of recent research [2-4]. In order to achieve these potential benefits, an adequate hard-decision detector for retrieving the information bits transmitted in the spatial and signal

*Correspondence: chengyp2000@vip.sina.com

College of Communications Engineering, PLA University of Science and

Technology, Nanjing, China constellation diagrams is needed. A matched filter (MF) detection was first proposed in [1], which detects the antenna index and modulated symbol separately. In [5], the authors first investigated the optimum hard-output maximum-likelihood (ML) detector, which jointly detects the transmit antenna index and the modulated symbol. To achieve a better trade-off between the performance and computational complexity, various sub-optimal hardoutput detectors based on ML and MF have been broadly investigated in [6-9], respectively.

Recently, to reduce the impact of channel fading and noise on bit error ratio (BER) performance, SM-MIMO systems with powerful channel coding, such as turbo codes and low-density parity-check (LDPC) codes, have gained rekindled interests [10-13]. A novel trellis coded spatial modulation (TCSM) scheme was proposed in

\section{Springer}

(c) $2016 \mathrm{Li}$ et al. Open Access This article is distributed under the terms of the Creative Commons Attribution 4.0 International License (http://creativecommons.org/licenses/by/4.0/), which permits unrestricted use, distribution, and reproduction in any medium, provided you give appropriate credit to the original author(s) and the source, provide a link to the Creative Commons license, and indicate if changes were made. 
[10], where the concept of trellis coded modulation was applied to the spatial constellation of SM systems. While it achieves better performance than that of uncoded SM system over correlated channels, it performs even worse in uncorrelated channels. To circumvent the problem, a novel MIMO transmission scheme was developed in [11], where a trellis encoder and a SM mapper are jointly designed to take advantage of the benefits of both. In [12], the authors designed a spectral efficiency transmission scheme, labeled as bit-interleaved coded spatial modulation (BICSM) with iterative demodulating/decoding, which provides substantial performance gains in all channel conditions. In [13], LDPC codes were optimized to match SM-MIMO systems based on the extrinsic information transfer chart technique. Although these prior works have significantly improved the understanding of error performance of coded SM-MIMO systems, the key limitation is that all of them employ the optimum maximum a posteriori probability (MAP) soft-output detector, the complexity of which is intractable when the number of transmit antennas is large or the modulation order is high. Hence, it is urgent to design low-complexity soft-output detectors for coded SM-MIMO systems.

Motivated by this observation, in a recent work [9], a low-complexity soft-output distance-based ordered detector (SODBD) was proposed; however, it significantly suffers from performance degradation compared to the optimal MAP soft-output detector. In addition, [14] investigated the soft-decision detectors conceived for SM and STSK systems, where the active antenna index and the modulated symbol are separately detected, respectively. Different from the prior works, in this paper, we shed a light on a universal low-complexity soft-decision aided detection algorithms for coded SM-MIMO systems. The main contributions of this paper are summarized as follows:

- For coded SM-MIMO systems with Gray-labeled phase-shift keying (PSK), we first design a new calculation of log-likelihood ratio (LLR) method based on the hard-decision detector in [6], denoted as PSK-based soft-output detector (PBSD) algorithm. The analytical results demonstrate that the PBSD algorithm significantly reduces the searched signal candidates from $N_{t} M$ to $N_{t}$, and the computational complexity of calculating the LLR of each bit is independent of the constellation size.

- For coded SM-MIMO systems with Gray-labeled quadrature amplitude modulation (QAM) modulation, we propose a low-complexity soft-output detector based on hard-limiter-based ML method proposed in [7], labeled as QAM-based softoutput detector (QBSD) algorithm. Compared to the Max-Log-LLR algorithm, it has much lower computational complexity at the expense of performance loss.

- Another contribution of this paper is that we further improve the above two soft-output detectors by adding a counterpart ML estimate to calculate the corresponding LLRs, namely, improved PSK-based soft-output detector (IPBSD) and improved QAM-based soft-output detector (IQBSD) algorithms, respectively. The analytical results, along with numerical analysis, reveal that both of them achieve the same performance as Max-Log-LLR algorithm with reduced computational complexity.

The rest of the paper is organized as follows. Section 2 introduces the coded SM-MIMO system model. Section 3 presents a brief overview of the existing detectors conceived for coded SM-MIMO systems. In Section 4, the proposed low-complexity soft-output detectors are presented. In Section 5, a comprehensive analysis of computational complexity for all the detectors is provided. Simulation results are presented in Section 6, and we make a conclusion in Section 7.

Notations. Upper/lower case bold symbols denote matrices/vectors. $(\cdot)^{H},(\cdot)^{T},(\cdot)^{*}$, and $(\cdot)^{\dagger}$ represent the hermitian transpose, transpose, complex conjugate and pseudo inverse of a vector, respectively. $p(a \mid b)$ denotes the probability density function (pdf) of random variable $a$ conditioned on $b, \operatorname{Pr}(\cdot)$ represents the probability of a variable. $\oplus$ denotes the bitwise XOR operation. $\Re(\cdot)$ and $\Im(\cdot)$ denote the real and imaginary parts of a complexvalued quantity, respectively. round $(a)$ is the operator that rounds the element $a$ to its nearest integer. $\bmod (a, n)$ denotes that $a$ is computed modulus $n$.

\section{Coded SM system model}

Let us consider a coded SM-MIMO system with $N_{t}$ transmit antennas and $N_{r}$ receive antennas, which is depicted in Fig. 1. The transmitter side consists of three parts, that is a channel encoder, a serial-parallel converter, and a SM mapper. The input source bits $\mathbf{u}$ is first encoded by a channel encoder with code rate $R$. Then, the output sequences of encoder $\mathbf{c}$ are converted from serial to parallel and then are utilized to modulate the SM symbol. We consider the conventional SM system, where the number of transmit antennas is an integer power of 2, i.e., $N_{t}=2^{Q_{1}}$, and $M$-ary digital modulation is employed with $M=2^{Q_{2}}$. Hence, at each time slot, each block consisting of $Q_{1}+Q_{2}$ bits are separated into two parts by SM mapper. The first part $Q_{1}$ bits, i.e., $\mathbf{c}_{A}$, are used to select the active antenna index from the spatial diagram, i.e., $\mathcal{S}=\left\{1,2, \cdots, N_{t}\right\}$, while the second part $Q_{2}$ bits, i.e., $\mathbf{c}_{S}$, are used to map the modulated signal from the symbol constellation, i.e., 

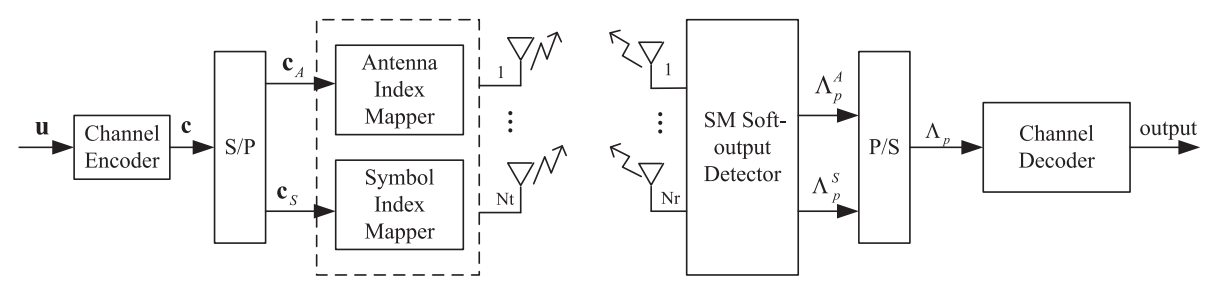

Fig. 1 Block diagram of coded SM-MIMO system

$\mathcal{M}=\left\{s_{0}, s_{1}, \cdots, s_{M-1}\right\}$. Hence, the transmit signal vector is given by

$$
\mathbf{x}=\left[0, \cdots, s_{q}, \cdots, 0\right]^{T} \in \mathcal{C}^{N_{t} \times 1}
$$

which implies that the $q$ th modulated symbol from the constellation $\mathcal{M}$ is transmitted from the $j$ th transmit antenna. Taking into account of the code rate $R$, the total spectral efficiency is $\eta=R\left(Q_{1}+Q_{2}\right)$ bits per channel use.

Assuming a quasi-static frequency flat fading channel, the received signal vector for coded SM-MIMO systems can be expressed as

$$
\mathbf{y}=\mathbf{H x}+\mathbf{n}=\mathbf{h}_{j} s_{q}+\mathbf{n},
$$

where $\mathbf{H}=\left[\mathbf{h}_{1}, \mathbf{h}_{2}, \ldots, \mathbf{h}_{N_{t}}\right] \in \mathcal{C}^{N_{r}} \times N_{t}$ denotes the channel matrix, and $\mathbf{h}_{j}$ is the $j$-th column of $\mathbf{H}$. Each entry in $\mathbf{H}$, i.e., $h_{i j}, i \in\left\{1, \ldots, N_{r}\right\}, j \in\left\{1, \ldots, N_{t}\right\}$, is zero mean and unit variance complex Gaussian random variable. $\mathbf{n}$ is a zero-mean additive white Gaussian noise vector with $\mathbb{E}\left[\mathbf{n n}^{H}\right]=\sigma^{2} \mathbf{I}_{N_{r} \times N_{r}}$.

At the receiver, to achieve the advantage of channel coding, a soft-output detector is employed, where the soft information for each coded bits is calculated based on the received signal. In coded SM-MIMO systems, the soft information consists of two parts, i.e., the soft information of antenna index bits and the soft information of modulated symbol bits. For the Log-MAP demapper, the soft information bits can be expressed in the form of the log-likelihood ratio (LLR). Without loss of generality, we define $\Lambda_{p}^{A}$ and $\Lambda_{p}^{S}$ as the a posterior LLRs of antenna index bits and modulated symbol bits, respectively.

\section{Conventional soft-decision aided SM detectors}

Before introducing our proposed low-complexity softdecision detectors, we first briefly summarize the conventional soft-output detectors for coded SM-MIMO systems, which are studied in Section 5 and Section 6 as a benchmark for assessing the performance versus computational complexity trade-off of the proposed soft-output detectors.
Different from the hard-decision detectors which only estimate the active antenna index and the modulated symbol, the soft-output detectors also compute the reliability of the estimation. Unless otherwise specified, we define $\mathcal{S}_{i}^{0}$ and $\mathcal{S}_{i}^{1}$ as the antenna index subsets, in which the $i$ th bit of each element is 0 and 1 , respectively. Similarly, $\mathcal{M}_{i}^{0}$ and $\mathcal{M}_{i}^{1}$ denote the modulated symbol subsets where the $i$ th bit of each element is 0 and 1 , respectively. Hence, for the soft-decision aided detection, the classic Log-MAP algorithm is given by [12]

$$
\begin{aligned}
\Lambda_{p}^{A}\left(c_{A, i}\right) & =\ln \frac{p\left(c_{A, i}=0 \mid \mathbf{y}\right)}{p\left(c_{A, i}=1 \mid \mathbf{y}\right)} \\
& =\ln \frac{\sum_{j \in \mathcal{S}_{i}^{0}} \sum_{s_{q} \in \mathcal{M}} p\left(\mathbf{h}_{j} \mid \mathbf{y}, s_{q}\right)}{\sum_{j \in \mathcal{S}_{i}^{1}} \sum_{s_{q} \in \mathcal{M}} p\left(\mathbf{h}_{j} \mid \mathbf{y}, s_{q}\right)} \\
& =\ln \frac{\sum_{j \in \mathcal{S}_{i}^{0}} \sum_{s_{q} \in \mathcal{M}} p\left(\mathbf{y} \mid \mathbf{h}_{j}, s_{q}\right) \operatorname{Pr}\left(\mathbf{h}_{j}\right)}{\sum_{j \in \mathcal{S}_{i}^{1}} \sum_{s_{q} \in \mathcal{M}} p\left(\mathbf{y} \mid \mathbf{h}_{j}, s_{q}\right) \operatorname{Pr}\left(\mathbf{h}_{j}\right)} \\
& =\ln \frac{\sum_{j \in \mathcal{S}_{i}^{0}} \sum_{s_{q} \in \mathcal{M}} p\left(\mathbf{y} \mid \mathbf{h}_{j}, s_{q}\right) \prod_{n=1}^{Q_{1}} \operatorname{Pr}\left(c_{A, n}\right)}{\sum_{j \in \mathcal{S}_{i}^{1}} \sum_{s_{q} \in \mathcal{M}} p\left(\mathbf{y} \mid \mathbf{h}_{j}, s_{q}\right) \prod_{n=1}^{Q_{1}} \operatorname{Pr}\left(c_{A, n}\right)}
\end{aligned}
$$

and

$$
\begin{aligned}
\Lambda_{p}^{S}\left(c_{S, i}\right) & =\ln \frac{p\left(c_{S, i}=0 \mid \mathbf{y}\right)}{p\left(c_{S, i}=1 \mid \mathbf{y}\right)} \\
& =\ln \frac{\sum_{s_{q} \in \mathcal{M}_{i}^{0}} \sum_{j \in \mathcal{S}} p\left(s_{q} \mid \mathbf{y}, \mathbf{h}_{j}\right)}{\sum_{s_{q} \in \mathcal{M}_{i}^{1}} \sum_{j \in \mathcal{S}} p\left(s_{q} \mid \mathbf{y}, \mathbf{h}_{j}\right)} \\
& =\ln \frac{\sum_{s_{q} \in \mathcal{M}_{i}^{0}} \sum_{j \in \mathcal{S}} p\left(\mathbf{y} \mid \mathbf{h}_{j}, s_{q}\right) \operatorname{Pr}\left(s_{q}\right)}{\sum_{s_{q} \in \mathcal{M}_{i}^{1}} \sum_{j \in \mathcal{S}} p\left(\mathbf{y} \mid \mathbf{h}_{j}, s_{q}\right) \operatorname{Pr}\left(s_{q}\right)} \\
& =\ln \frac{\sum_{s_{q} \in \mathcal{M}_{i}^{0}} \sum_{j \in \mathcal{S}} p\left(\mathbf{y} \mid \mathbf{h}_{j}, s_{q}\right) \prod_{n=1}^{Q_{2}} \operatorname{Pr}\left(c_{S, n}\right)}{\sum_{s_{q} \in \mathcal{M}_{i}^{1}} \sum_{j \in \mathcal{S}} p\left(\mathbf{y} \mid \mathbf{h}_{j}, s_{q}\right) \prod_{n=1}^{Q_{2}} \operatorname{Pr}\left(c_{S, n}\right)},
\end{aligned}
$$


where $\Lambda_{p}^{A}\left(c_{A, i}\right)$ and $\Lambda_{p}^{S}\left(c_{S, i}\right)$ represent the a posteriori LLRs for the $i$ th antenna index bit $c_{A, i}$ and $i$ th modulated symbol bit $c_{S, i}$, respectively, $\operatorname{Pr}\left(\mathbf{h}_{j}\right)=\prod_{n=1}^{Q_{1}} \operatorname{Pr}\left(c_{A, n}\right)$ and $\operatorname{Pr}\left(s_{q}\right)=\prod_{n=1}^{Q_{2}} \operatorname{Pr}\left(c_{S, n}\right)$ are the a prior probability of the antenna index and the modulated signal, respectively, $\operatorname{Pr}\left(c_{A, n}\right)$ and $\operatorname{Pr}\left(c_{S, n}\right)$ denote the a prior probability of the $n$-th bits of the antenna index and modulated symbol, respectively, and

$$
p\left(\mathbf{y} \mid \mathbf{h}_{j}, s_{q}\right)=\frac{1}{\left(2 \pi \sigma^{2}\right)^{N_{r} / 2}} \exp \left(-\frac{\left\|\mathbf{y}-\mathbf{h}_{j} s_{q}\right\|^{2}}{2 \sigma^{2}}\right) .
$$

By substituting Eq. (5) into Eq. (3) and Eq. (4), we have

$$
\Lambda_{p}^{A}\left(c_{A, i}\right)=\ln \frac{\sum_{j \in \mathcal{S}_{i}^{0}} \sum_{s_{q} \in \mathcal{M}} \exp \left(-\left\|\mathbf{y}-\mathbf{h}_{j} s_{q}\right\|^{2} / 2 \sigma^{2}\right)}{\sum_{j \in \mathcal{S}_{i}^{1}} \sum_{s_{q} \in \mathcal{M}} \exp \left(-\left\|\mathbf{y}-\mathbf{h}_{j} s_{q}\right\|^{2} / 2 \sigma^{2}\right)}
$$

and

$$
\Lambda_{p}^{S}\left(c_{S, i}\right)=\ln \frac{\sum_{s_{q} \in \mathcal{M}_{i}^{0}} \sum_{j \in \mathcal{S}} \exp \left(-\left\|\mathbf{y}-\mathbf{h}_{j} s_{q}\right\|^{2} / 2 \sigma^{2}\right)}{\sum_{s_{q} \in \mathcal{M}_{i}^{1}} \sum_{j \in \mathcal{S}} \exp \left(-\left\|\mathbf{y}-\mathbf{h}_{j} s_{q}\right\|^{2} / 2 \sigma^{2}\right)} .
$$

To avoid the complex exponential and logarithmic operations in the Log-MAP algorithm, we can resort to the simplified Max-Log-LLR algorithm as [15]

$$
\begin{array}{r}
\Lambda_{p}^{A}\left(c_{A, i}\right)=\frac{1}{2 \sigma^{2}}\left[\min _{j \in \mathcal{S}_{i}^{1}, s_{q} \in \mathcal{M}}\left(\left\|\mathbf{y}-\mathbf{h}_{j} s_{q}\right\|^{2}\right)\right. \\
\left.-\min _{j \in \mathcal{S}_{i}^{0}, s_{q} \in \mathcal{M}}\left(\left\|\mathbf{y}-\mathbf{h}_{j} s_{q}\right\|^{2}\right)\right]
\end{array}
$$

and

$$
\begin{array}{r}
\Lambda_{p}^{S}\left(c_{S, i}\right)=\frac{1}{2 \sigma^{2}}\left[\min _{s_{q} \in \mathcal{M}_{i}^{1}, j \in \mathcal{S}}\left(\left\|\mathbf{y}-\mathbf{h}_{j} s_{q}\right\|^{2}\right)\right. \\
\left.-\min _{s_{q} \in \mathcal{M}_{i}^{0}, j \in \mathcal{S}}\left(\left\|\mathbf{y}-\mathbf{h}_{j} s_{q}\right\|^{2}\right)\right] .
\end{array}
$$

Note that the computational complexity of Max-LogLLR algorithm has been significantly reduced; however, the number of multiplications and comparisons remains high, which is the order of $O\left(M N_{t}\right)$.

\section{The proposed low-complexity soft-decision aided SM detectors}

In this section, we present our new low-complexity softoutput detectors in detail. Four different low-complexity soft-decision aided detectors are proposed, which are based on the following zero-forcing (ZF) output, that is,

$$
r_{j}=\mathbf{h}_{j}^{\dagger} \mathbf{y}
$$

where $\mathbf{h}_{j}^{\dagger}=\frac{\mathbf{h}_{j}^{H}}{\mathbf{h}_{j}^{H} \mathbf{h}_{j}}$ and $j \in\left\{1,2, \cdots, N_{t}\right\}$.

\subsection{Low-complexity soft-output SM detectors with Gray-labeled M-PSK}

In this subsection, we propose two low-complexity softoutput detectors for coded SM-MIMO systems with Gray-labeled $M$-PSK. For easy illustration, we take the 8PSK as an example; however, the proposed two algorithms are also adapted for arbitrary $M$-PSK modulation. The 8PSK constellation is illustrated in Fig. 2, where the initial phase of the constellation is $\varphi$, then the $k$ th constellation point of $M$-PSK can be expressed as

$$
s_{k}=\exp \left(J\left(\frac{2 \pi}{M} k+\varphi\right)\right)
$$

where $J=\sqrt{-1}$ and $k \in\{0,1, \cdots, M-1\}$.

\subsubsection{PBSD algorithm}

As indicated in [6], the optimal ML detection for SMMIMO systems is given by

$$
\left[\widehat{j}, \widehat{s}_{k}\right]=\arg \min _{j \in \mathcal{S}}\left(\min _{s_{k} \in \mathcal{M}}\left(\left\|\mathbf{y}-\mathbf{h}_{j} s_{k}\right\|^{2}\right)\right) .
$$

By substituting Eq. (10) into Eq. (12) and performing some simple manipulations, the inner minimization in Eq. (12) can be further expressed as

$$
\widehat{s}_{k}=\arg \min _{s_{k} \in \mathcal{M}}\left(\left\|r_{j}-s_{k}\right\|^{2}\right)
$$

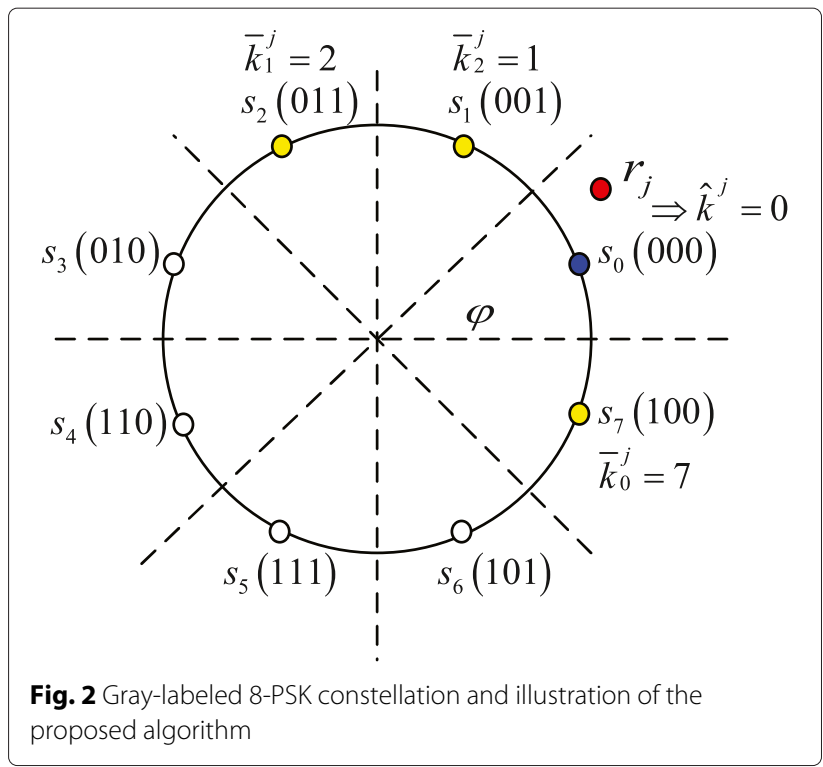


Now, by substituting the polar coordinate form of $r_{j}=$ $\left|r_{j}\right| \exp \left(J \theta_{j}\right)$ and Eq. (11) into Eq. (13), we have

$$
\begin{aligned}
\widehat{k}^{j} & =\arg \min _{k}\left(\left|r_{j}\right|^{2}+1-2\left|r_{j}\right| \cos \left(\theta_{j}-\left(k \frac{2 \pi}{M}+\varphi\right)\right)\right) \\
& =\arg \max _{k}\left(\cos \left(\theta_{j}-\left(k \frac{2 \pi}{M}+\varphi\right)\right)\right),
\end{aligned}
$$

where $\widehat{k}^{j}$ represents the index of the constellation signal nearest to the received signal $r_{j}$ for the $j$ th antenna (shown in Fig. 2). Hence, by exploiting the symmetric features of $M$-PSK constellation, the estimated index of the modulated signal on the $j$ th antenna can be expressed as [8]

$$
\widehat{k}^{j}=\left\{\begin{array}{lr}
0, & \varphi-\frac{\pi}{M} \leq \theta_{j}<\varphi+\frac{\pi}{M} \\
1, & \varphi+\frac{\pi}{M} \leq \theta_{j}<\varphi+3 \frac{\pi}{M} \\
\vdots & \varphi+(M-1.5) \frac{2 \pi}{M} \leq \theta_{j} \\
M-1, & <\varphi+(M-0.5) \frac{2 \pi}{M}
\end{array}\right.
$$

Thus, the relationship between the phase of the received signal and the index of the modulated symbol can be concluded as

$$
\widehat{k}^{j}=\bmod \left(\operatorname{round}\left(\frac{\theta_{j}-\varphi}{2 \pi / M}\right), M\right) .
$$

As a result, the estimated signal is given by

$$
s_{\widehat{k}^{j}}=\exp \left(J\left(\widehat{k}^{j} * \frac{2 \pi}{M}+\varphi\right)\right) .
$$

Before introducing our proposed PBSD algorithm, we first define $\mathcal{X}$ as the collect of $s_{\widehat{k}}$, i.e., $\mathcal{X}=$ $\left\{s_{\widehat{k}^{1}}, s_{\widehat{k}^{2}}, \cdots, \widehat{s}_{\mathrm{k}^{N}}\right\}$. Different from the Max-Log LLR algorithm, the element of the signal candidate list is diminished in the PBSD algorithm. In doing so, we first utilize (16) and (17) to estimate the modulated signal for each transmit antenna. Hence, the length of the signal candidate list is reduced from $N_{t} M$ to $N_{t}$. In addition, the following equation

$$
\left\|\mathbf{y}-\mathbf{h}_{j} s_{\widehat{k}^{j}}\right\|^{2}=\|\mathbf{y}\|^{2}+\left\|\mathbf{h}_{j}\right\|^{2}\left[1-2 \Re\left(r_{j} s_{\widehat{k}^{j}}^{*}\right)\right]
$$

holds. Thus, by substituting Eq. (18) into Eq. (8) and Eq. (9), and limiting the searched signal space to $\mathcal{X}$, the corresponding LLRs for the $i$ th antenna index bit and the modulated symbol bit under the proposed PBSD algorithm are given by.

$$
\begin{aligned}
\Lambda_{p}^{A}\left(c_{A, i}\right)=\frac{1}{2 \sigma^{2}} & {\left[\min _{j \in \mathcal{S}_{i}^{1}}\left(\left\|\mathbf{h}_{j}\right\|^{2}\left(1-2 \Re\left(r_{j} s_{\widehat{k}^{j}}^{*}\right)\right)\right)\right.} \\
& \left.-\min _{j \in \mathcal{S}_{i}^{0}}\left(\left\|\mathbf{h}_{j}\right\|^{2}\left(1-2 \Re\left(r_{j} s_{\widehat{k}^{j}}^{*}\right)\right)\right)\right]
\end{aligned}
$$

and

$$
\begin{aligned}
\Lambda_{p}^{S}\left(c_{S, i}\right)= & \frac{1}{2 \sigma^{2}}\left[\min _{s_{\widehat{k}^{j}} \in \mathcal{X}_{i}^{1}}\left(\left\|\mathbf{h}_{j}\right\|^{2}\left(1-2 \Re\left(r_{j} s_{\widehat{k}^{j}}^{*}\right)\right)\right)\right. \\
& \left.-\min _{s_{\widehat{k}^{j}} \in \mathcal{X}_{i}^{0}}\left(\left\|\mathbf{h}_{j}\right\|^{2}\left(1-2 \Re\left(r_{j} s_{\hat{k}^{j}}^{*}\right)\right)\right)\right] .
\end{aligned}
$$

\subsubsection{IPBSD algorithm}

As discussed in the above, when computing the LLRs of the modulated symbol bits, the proposed PBSD algorithm only finds the ML estimate of the symbol and ignores the counterpart ML candidate whose $i$ th bit is contrary to the ML estimate. Therefore, this results in the performance gap between the proposed PBSD algorithm and the Max-Log-LLR algorithm. In this subsection, we propose a new improved algorithm based on the PBSD algorithm, namely, IPBSD algorithm, by taking the counterpart ML estimate into account when computing the LLRs of the modulated symbol bits.

By careful inspection of Eq. (9), we find that the item $\min _{q \in \mathcal{M}, j \in \mathcal{S}}\left\|\mathbf{y}-\mathbf{h}_{j} s_{q}\right\|^{2}$, i.e., the squared Euclidean distance from the received signal vector $\mathbf{y}$ to the nearest constellation point $s_{\widehat{k}^{j}}$ for the $j$ th antenna, always appears in Eq. (9). It is equal to either $\min _{s_{q} \in \mathcal{M}_{i}^{0}, j \in \mathcal{S}}\left\|\mathbf{y}-\mathbf{h}_{j} s_{q}\right\|^{2}$ or $\min _{s_{q} \in \mathcal{M}_{i}^{1}, j \in \mathcal{S}}\left\|\mathbf{y}-\mathbf{h}_{j} s_{q}\right\|^{2}$, which depends on the $i$ th bit of $s_{\widehat{k}^{j}}$ being 0 or 1 . Hence, our aim is to find another signal point $s_{\bar{k}_{i}^{j}}$ resulting in the other minimum term in Eq. (9), which means that the $i$ th bit of $s_{\bar{k}_{i}^{j}}$ is opposite to the $i$ th bit of $s_{\widehat{k}^{j}}$. Moreover, as shown in Fig. 2 , if the antenna index is $j$, the nearest signal point to the ZF output $r_{j}$ is $s_{0}$, the index of which is $\widehat{k}^{j}=0$. Hence, the first bit of $s_{0}$ is 0 , and the nearest signal point to $r_{j}$ with the first bit being 1 is $s_{7}$, that is, $\bar{k}_{0}^{j}=7$. Similarly, for the second and third bits, we have $\bar{k}_{1}^{j}=2$ and $\bar{k}_{2}^{j}=1$, respectively.

Before introducing the detailed procedure of the proposed IPBSD algorithm, we first present the following lemmas for binary-reflected Gray-labeled constellations [16].

Lemma 1. For binary-reflect Gray Labeling $\mathbf{g}^{k} \rightarrow s_{k}$, by denoting $\mathbf{b}^{k}=\left(b_{0}^{k}, b_{1}^{k}, \cdots, b_{Q_{2}-1}^{k}\right)$ as the binary representation of the symbol index $k$ with the most significant bit (MSB) as the leftmost bit, then, $\mathbf{g}^{k}$ is given by

$$
\begin{aligned}
\mathbf{g}^{k} & =\left(g_{0}^{k}, g_{1}^{k}, \cdots, g_{Q_{2}-1}^{k}\right) \\
& =\left(b_{0}^{k}, b_{1}^{k}, \cdots, b_{Q_{2}-1}^{k}\right) \oplus\left(0, b_{0}^{k}, \cdots, b_{Q_{2}-2}^{k}\right) .
\end{aligned}
$$


Lemma 2. For the binary-reflected Gray PSK $\widehat{\mathbf{g}}^{\widehat{k}^{j}} \rightarrow s_{\widehat{k}^{j}}$, where $s_{\widehat{k}^{j}}$ is the nearest signal point to the filtered signal $r_{j}$, $\mathbf{b}^{\widehat{k}^{j}}=\left(b_{0}^{k^{j}}, b_{1}^{\widehat{k}^{j}}, \cdots, b_{Q_{2}-1}^{\widehat{k}^{j}}\right)$ is the binary representation of $\widehat{k}^{j}$ with the MSB as the leftmost bit. Let $\mathcal{M}_{i}^{\widehat{\bar{g}}_{i}^{k^{j}}}$ denote the constellation point subset where the ith bit of each point is contrary to the bit $\widehat{g}_{i}^{k^{j}}$, i.e., $\bar{g}_{i}^{k^{j}}=1-g_{i}^{k^{j}}$, then the index of the nearest constellation point to $r_{j}$ in subset $\mathcal{M}_{i}^{\bar{g}_{i}^{\hat{k}^{j}}}$ can be determined as

$$
\bar{k}_{i}^{j}= \begin{cases}\bar{b}_{0}^{k^{j}} 2^{Q_{2}-1}+\bar{b}_{1}^{\widehat{k}^{j}}\left(2^{Q_{2}-1}-1\right) & i=0 \\ 2^{Q_{2}-i-1}-b_{i}^{\widehat{k}^{j}}+\sum_{n=0}^{i-1} b_{n}^{\widehat{k}^{j}} 2^{Q_{2}-n-1} & i>0\end{cases}
$$

where $\bar{b}_{i}^{\widehat{k}^{j}}=1-b_{i}^{\widehat{k}^{j}}$.

Proof: The proof can be found in [16].

It is noted from Lemma 1 that for the $i$ th bit of the modulated symbol, the candidate list $\mathcal{W}_{i}=$ $\left\{s_{\widehat{k}^{1}}, \cdots, s_{\widehat{k}^{N_{t}}}, s_{\bar{k}_{i}^{1}}, \cdots, s_{\bar{k}_{i}^{N_{t}}}\right\}$ can be obtained without any comparison. To this end, the detailed procedure of the proposed IPBSD algorithm can be described as follows.

Step 1: The first step is to compute the ZF filtered signal $r_{j}$ for each transmit antenna based on Eq. (10).

Step 2: According to Eq. (16) and Eq. (17), the second step is to find the index of the modulated signal $\widehat{k}^{j}$ and the estimated modulated symbol $s_{\widehat{k}^{j}}$. Afterwards, the binary representation $\widehat{\mathbf{b}}^{\hat{k}^{j}}$ of $\widehat{k}^{j}$ can be achieved, and thus, we can get the Gray labeling representation $\mathbf{g}^{\widehat{k}^{j}}$ from Eq. (21).

Step 3: After performing Step 2, we have obtained the estimated signal symbol, which results in both minimum terms in Eq. (8), while only one of the minimum term in Eq. (9). Hence, in this step, we need to find the signal points that result in another minimum term of Eq. (9) based on Lemma 2.

Step 4: Finally, the a posteriori LLR of the modulated symbol bits can be derived as

$$
\begin{array}{r}
\Lambda_{p}^{S}\left(c_{S, i}\right)=\frac{1}{2 \sigma^{2}}\left[\min _{s \in \mathcal{W}_{i}^{1}}\left(\left\|\mathbf{h}_{j}\right\|^{2}\left(1-2 \Re\left(r_{j} s^{*}\right)\right)\right)\right. \\
\left.-\min _{s \in \mathcal{W}_{i}^{0}}\left(\left\|\mathbf{h}_{j}\right\|^{2}\left(1-2 \Re\left(r_{j} s^{*}\right)\right)\right)\right]
\end{array}
$$

while the a posteriori LLR of the antenna index bits is the same as the proposed PBSD algorithm in Eq. (19).

\subsection{Low-complexity soft-output SM detector with Gray-labeled $M$-QAM}

In this subsection, we design two low-complexity softoutput detectors for coded SM-MIMO systems with square or a rectangular QAM modulation, called QBSD and IQBSD algorithms. As pointed out in [17], a lattice constellation, e.g., square or rectangular QAM, can be regraded as a Cartesian product of two independent PAM signal sets, say $N_{1}$-PAM and $N_{2}$-PAM, where $N_{1}$ and $N_{2}$ represent the number of in-phase and quadraturephase points, respectively. Hence, the proposed QBSD and IQBSD algorithms are based on the two PAM constellations instead of the QAM. Without loss of generality, we take the 16-QAM for example, the first two bits "10" of the signal point " 1001 " determine the real part of the signal symbol, while the last two bits "01" decide the imaginary part. The in-phase PAM constellation of 16-QAM and the illustration of the proposed algorithm are shown in Fig. 3, where $\Re\left(r_{j}\right)$ denotes the real part of the filtered signal $r_{j}$.

\subsubsection{QBSD algorithm}

In the QBSD algorithm, we first estimated the modulated symbol based on the hard-decision detector in [7], from which the optimal ML detection can be equivalently expressed as

$$
\begin{aligned}
{\left[\widehat{j}, \widehat{s}_{q}\right] } & =\arg \min _{j \in \mathcal{S}}\left(\min _{s \in \mathcal{M}}\left(\left|r_{j}-s\right|^{2}\right)\right) \\
& =\arg \min _{j \in \mathcal{S}}\left(\min _{s_{I} \in N_{1}-\mathrm{PAM}, s_{Q} \in N_{2}-\mathrm{PAM}}\left(\left(\Re\left(r_{j}\right)-s_{I}\right)^{2}+\left(\Im\left(r_{j}\right)-s_{Q}\right)^{2}\right)\right) .
\end{aligned}
$$

Since $s_{I}$ and $s_{Q}$ are from orthogonal dimensions, the inner minimizations in Eq. (24) can be decomposed into two optimization problems, that is,

$$
\Re\left(\widehat{s}^{j}\right)=\min _{s_{I} \in N_{1}-\mathrm{PAM}}\left(\Re\left(r_{j}\right)-s_{I}\right)^{2}
$$

and

$$
\mathfrak{s}\left(\widehat{s}^{j}\right)=\min _{s_{Q} \in N_{2}-\mathrm{PAM}}\left(\mathfrak{s}\left(r_{j}\right)-s_{Q}\right)^{2}
$$

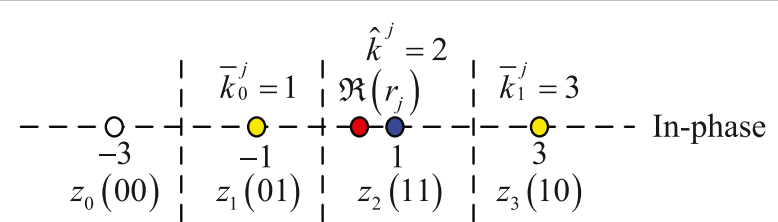

Fig. 3 Gray-labeled 4-PAM constellation and illustration of the proposed algorithm 
As the symbols of $N_{1}$-PAM are given by $\{2 t+1\}_{t=\frac{-N_{1}}{2}}^{\frac{N_{1}}{2}-1}$, $\Re(\widehat{S})$ can be derived as follows:

$$
\begin{array}{cc}
\Re\left(r_{j}\right)<-N_{1}+2 \Rightarrow & \Re\left(\widehat{s}^{j}\right)=-N_{1}+1 \\
-N_{1}+2 \leq \Re\left(r_{j}\right)<-N_{1}+4 \Rightarrow & \Re\left(\widehat{s}^{j}\right)=-N_{1}+3 \\
\vdots & \vdots \\
-2 \leq \Re\left(r_{j}\right)<0 \Rightarrow & \Re\left(\widehat{s}^{j}\right)=-1 \\
0 \leq \Re\left(r_{j}\right)<2 \Rightarrow & \Re\left(\widehat{s}^{j}\right)=1 \\
\vdots & \vdots \\
N_{1}-4 \leq \Re\left(r_{j}\right)<N_{1}-2 \Rightarrow & \Re\left(\widehat{s}^{j}\right)=N_{1}-3 \\
N_{1}-2 \leq \Re\left(r_{j}\right) \Rightarrow & \Re\left(\widehat{s}^{j}\right)=N_{1}-1
\end{array}
$$

However, according to [7], $\Re\left(\widehat{s}^{j}\right)$ can be obtained directly as (27) without employing the above set of comparisons.

$\Re\left(\widehat{s}^{j}\right)=\min \left(\max \left(2 * \operatorname{round}\left(\frac{\Re\left(r_{j}\right)+1}{2}\right)-1,-N_{1}+1\right), N_{1}-1\right)$

Similarly, $\Im\left(\widehat{s}^{j}\right)$ can also be obtained directly as

$\Im\left(\widehat{s}^{j}\right)=\min \left(\max \left(2 * \operatorname{round}\left(\frac{\Im\left(r_{j}\right)+1}{2}\right)-1,-N_{2}+1\right), N_{2}-1\right)$.

Thus, the estimated signal is expressed as

$$
\widehat{s}^{j}=\Re\left(\widehat{s}^{j}\right)+J \Im\left(\widehat{s}^{j}\right) .
$$

Let us define $\mathcal{U}=\left\{\widehat{s}^{1}, \widehat{s}^{2}, \cdots, \widehat{s}^{N_{t}}\right\}$, then, the corresponding LLRs of the $i$-th antenna index bit and modulated signal bit under the proposed QBSD detector are given by

$$
\begin{aligned}
\Lambda_{p}^{A}\left(c_{A, i}\right)= & \frac{1}{2 \sigma^{2}} \min _{j \in \mathcal{S}_{i}^{1}}\left(\left\|\mathbf{y}-\mathbf{h}_{j} \widehat{s}^{j}\right\|^{2}\right) \\
& -\frac{1}{2 \sigma^{2}} \min _{j \in \mathcal{S}_{i}^{0}}\left(\left\|\mathbf{y}-\mathbf{h}_{j} \widehat{s}^{j}\right\|^{2}\right) \\
= & \frac{1}{2 \sigma^{2}} \min _{j \in \mathcal{S}_{i}^{1}}\left(\left\|\mathbf{h}_{j}\right\|^{2}\left(\left|r_{j}-\widehat{s}^{j}\right|^{2}-\left|r_{j}\right|^{2}\right)\right) \\
& -\frac{1}{2 \sigma^{2}} \min _{j \in \mathcal{S}_{i}^{0}}\left(\left\|\mathbf{h}_{j}\right\|^{2}\left(\left|r_{j}-\widehat{s}_{j}\right|^{2}-\left|r_{j}\right|^{2}\right)\right)
\end{aligned}
$$

and

$$
\begin{aligned}
\Lambda_{p}^{S}\left(c_{S, i}\right)= & \frac{1}{2 \sigma^{2}} \min _{\widehat{s}^{j} \in \mathcal{U}_{i}^{1}}\left(\left\|\mathbf{y}-\mathbf{h}_{j} \widehat{s}^{j}\right\|^{2}\right) \\
& -\frac{1}{2 \sigma^{2}} \min _{\widehat{s}^{j} \in \mathcal{U}_{i}^{0}}\left(\left\|\mathbf{y}-\mathbf{h}_{j} \widehat{s}^{j}\right\|^{2}\right) \\
= & \frac{1}{2 \sigma^{2}} \min _{\widehat{s}^{j} \in \mathcal{U}_{i}^{1}}\left(\left\|\mathbf{h}_{j}\right\|^{2}\left(\left|r_{j}-\widehat{s}^{j}\right|^{2}-\left|r_{j}\right|^{2}\right)\right) \\
& -\frac{1}{2 \sigma^{2}} \min _{\widehat{s}^{j} \in \mathcal{U}_{i}^{0}}\left(\left\|\mathbf{h}_{j}\right\|^{2}\left(\left|r_{j}-\widehat{s}^{j}\right|^{2}-\left|r_{j}\right|^{2}\right)\right) .
\end{aligned}
$$

Noting from Eqs. (30) and (31), we find that the length of the candidate list of the proposed QBSD detector is reduced from $N_{t} M$ to $N_{t}$.

\subsubsection{IQBSD algorithm}

As the above discussion, the QBSD algorithm only calculates the ML estimated symbol, which results in one of the minimum of Max-Log-LLR algorithm in Eq. (9). Hence, the error performance of the QBSD algorithm is inferior to that of Max-Log-LLR algorithm. Against this observation, the aim of IQBSD algorithm is to eliminate the performance gap by searching the counterpart ML estimate.

As illustrated in Fig. 3, the ML estimate of the modulated signal is the point $z_{2}$ in the 4-PAM constellation, in which the first and second bits are both "1." Therefore, the constellation point with the first bit being 0 and nearest to the real part of $r_{j}$ is the signal point $z_{1}$ in the 4-PAM constellation. Moreover, the constellation point with the second bit being 0 and nearest to the real part of $r_{j}$ is the signal point $z_{3}$. Before describing the procedure of the IQBSD algorithm in detail, we first present the following lemma.

Lemma 3. Let $\mathcal{N}$ denote the set of $N_{1}-P A M$ constellation points, $\widehat{k}^{j}$ is the index of the estimated symbol $\Re\left(\widehat{s}^{j}\right)$ in the $N_{1}$-PAM constellation. $\mathcal{N}_{i}^{\bar{g}_{i}^{\hat{k}^{j}}}$ denotes the subset of the constellation points whose ith bit is contrary to the bit $\widehat{g}_{i}^{k^{j}}$, i.e., $\widehat{g}_{i}^{k^{j}}=1-{g_{i}}_{k^{j}}$. Then, the index of the point in subset $\mathcal{N}_{i}^{\vec{g}_{i}^{\vec{k} j}}$ nearest to $\Re\left(r_{j}\right)$ can be derived as

$$
\bar{k}_{i}^{j}=2^{m_{1}-i-1}-b_{i}^{\widehat{k}^{j}}+\sum_{n=0}^{i-1} \widehat{b}_{n}^{\widehat{k}^{j}} 2^{m_{1}-n-1},
$$

where $m_{1}=\log _{2}\left(N_{1}\right)$ and $i \in\left\{0,1, \cdots, m_{1}-1\right\}$.

Note that, if the bit belong to the imaginary part of the modulated symbol, we can only substitute $m_{1}$ with $m_{2}$ in Eq. (32), where $m_{2}=\log _{2}\left(N_{2}\right)$.

According to Lemma 3 and the ML estimated signal as QBSD algorithm, if the bit belong to the real part of the modulated symbol, then the candidate list for the $i$ th 
bit of modulated symbol can be represented as (33). On the other hand, if the bit belong to the imaginary part of the modulated symbol, the candidate list for the $i$ th bit of modulated symbol can be represented as (34).

$\mathcal{V}_{i}=\left\{\widehat{s}^{1}, \cdots, \widehat{s}^{N_{t}}, z_{\bar{k}_{i}^{1}}+J * \mathcal{I}\left(\widehat{s}^{1}\right), \cdots, z_{\bar{k}_{i} N_{t}}+J * \mathcal{I}\left(\widehat{s}^{N_{t}}\right)\right\}$.

$\mathcal{V}_{i}=\left\{\widehat{s}^{1}, \cdots, \widehat{s}^{N_{t}}, \Re\left(\widehat{s}^{1}\right)+J * z_{\bar{k}_{i}^{1}}, \cdots, \Re\left(\widehat{s}^{N_{t}}\right)+J * z_{\bar{k}_{i}}\right\}$.

Now, the detail process of the IQBSD algorithm will be discussed in the following. Please note that the following description is restricted to the real part of the signal; however, the process of estimating the imaginary part of the signal is similar.

Step 1: The first step of IQBSD algorithm is the same as the IPBSD algorithm, i.e., calculating the filter output $r_{j}$ for each antenna based on Eq. (10).

Step 2 After obtaining $r_{j}$, the symbol $\Re\left(\widehat{s}^{j}\right)$ in the $N_{1}$ PAM constellation nearest to $\Re\left(r_{j}\right)$ can be calculated by Eq. (27). $\widehat{k}^{j}$ is the index of the symbol $\Re\left(\widehat{s}^{j}\right)$ in $N_{1}$-PAM constellation, and the binary representation of the index $\widehat{k}^{j}$ is $\mathbf{b}^{\widehat{k}^{j}}=\left(b_{0}^{\widehat{k}^{j}}, b_{1}^{\widehat{k}^{j}}, \ldots, b_{m_{1}-1}^{\widehat{k}^{j}}\right)$. Then, the binary-reflected Gray labeling $\widehat{\mathbf{g}}^{\widehat{k}^{j}}$ can be achieved by Lemma 1 .

Step 3: The objective of this step is to find the counterpart ML estimate $z_{\bar{k}_{i}^{j}}$ for the $i$ th bit, where the corresponding index is denoted as $\bar{k}_{i}^{j}$. Considering the symmetric structure of Gray-labeled PAM, we can resort to Lemma 3 to find the signal $z_{\bar{k}_{i}^{j}}$, which only requires the binary representation of index $\widehat{k}^{j}$ and addition operation, instead of computing the Euclidean distance between all the signals with the $i$ th bit being opposite to that of $z_{\widehat{k}^{j}}$ and $\Re\left(r_{j}\right)$.

Step 4: Finally, the a posteriori LLR of the antenna index bits can be derived as Eq. (30), and the a posteriori LLR of the symbol bits is obtained as

$$
\begin{aligned}
\Lambda_{p}^{S}\left(c_{S, i}\right)= & \frac{1}{2 \sigma^{2}}\left[\min _{s^{\prime} \in \mathcal{V}_{i}^{1}}\left(s\left\|\mathbf{h}_{j}\right\|^{2}\left(\left|r_{j}-s^{\prime}\right|^{2}-\left|r_{j}\right|^{2}\right)\right)\right. \\
& \left.-\frac{1}{2 \sigma^{2}} \min _{s^{\prime} \in \mathcal{V}_{i}^{0}}\left(\left\|\mathbf{h}_{j}\right\|^{2}\left(\left|r_{j}-s^{\prime}\right|^{2}-\left|r_{j}\right|^{2}\right)\right)\right],
\end{aligned}
$$

where $j$ is the antenna index corresponding to the modulated symbol $s^{\prime}$ selected from the set $\mathcal{V}_{i}$ in Eq. (33).

\section{Complexity analysis}

In this section, the computational complexity of the proposed soft-decision aided detectors and the Max-Log-LLR detector will be discussed in detail. Without loss of generality, the computational complexity is evaluated in terms of the number of real multiplications, real additions, and comparisons, respectively.

\subsection{Max-Log-LLR algorithm}

The number of real multiplications and real additions for computing the term $\left\|\mathbf{y}-\mathbf{h}_{j} s_{q}\right\|^{2}$ is $6 N_{r}$ and $6 N_{r}-1$, respectively. While for the Max-Log-LLR algorithm, the term $\left\|\mathbf{y}-\mathbf{h}_{j} s_{q}\right\|^{2}$ needs to be calculated $N_{t} M$ times. In addition, the calculation of LLR for each bit needs one real multiplication and one real addition, respectively. Considering that the number of bits in each spatial modulation symbol is $\log _{2}\left(N_{t} M\right)$, hence, the total number of real multiplications and real additions are given by

$$
\begin{aligned}
& C_{\mathrm{M}}^{\mathrm{Max}}=6 N_{r} N_{t} M+\log _{2}\left(N_{t} M\right) \\
& C_{\mathrm{A}}^{\mathrm{Max}}=6 N_{r} N_{t} M+\log _{2}\left(N_{t} M\right)-N_{t} M .
\end{aligned}
$$

On the other hand, since the length of the candidate list is $N_{t} M$, we need to find the minimum among all $N_{t} M$ candidates for each bit. Hence, the total number of comparison operation is

$$
C_{\mathrm{C}}^{\mathrm{Max}}=N_{t} M \log _{2}\left(N_{t} M\right) .
$$

\subsection{PBSD algorithm}

The computation of Eq. (10) needs $6 N_{r}+2$ real multiplications and $6 N_{r}-2$ real additions, respectively. While the acquisition of the modulated symbol for each transmit antenna needs 2 real multiplications and 2 real additions, respectively. Calculating the minimum term, which appears in the equations of calculating the LLRs in Eq. (18), needs only 4 real multiplications and 2 real additions. All the above terms need to be computed by $N_{t}$ times; thus, the total computational complexity of real multiplications and real additions of PBSD algorithm is given by

$$
\begin{aligned}
& C_{\mathrm{M}}^{\mathrm{PBSD}}=6 N_{r} N_{t}+8 N_{t}+\log _{2}\left(N_{t} M\right) \\
& C_{\mathrm{A}}^{\mathrm{PBSD}}=6 N_{r} N_{t}+\log _{2}\left(N_{t} M\right)+2 N_{t} .
\end{aligned}
$$

On the other hand, as the length of the candidate list is $N_{t}$ and the comparison number of each bit is $N_{t}$, therefore, the total comparison number of PBSD algorithm is

$$
C_{\mathrm{C}}^{\mathrm{PBSD}}=N_{t} \log _{2}\left(N_{t} M\right) \text {. }
$$

\subsection{IPBSD algorithm}

Compared to the PBSD algorithm, IPBSD algorithm also provides the counterpart ML estimate for each modulated bit. Hence, the length of the candidate list for computing 
the antenna bits is $N_{t}$ as PBSD algorithm, while the length of the candidate list for computing the modulated bits is $2 N_{t}$ under the worst case. In addition, the computational complexity of calculating the index of the counterpart ML estimate from Eq. (22) can be neglected due to it needs only a few additions. Hence, the computational complexity of real multiplications, real additions, and comparisons for the IPBSD algorithm is given by

$$
\begin{aligned}
& C_{\mathrm{M}}^{\mathrm{IPBSD}}=6 N_{r} N_{t}+8 N_{t}+\log _{2}\left(N_{t} M\right)+5 N_{t} \log _{2}(M) \\
& C_{\mathrm{A}}^{\mathrm{IPBSD}}=6 N_{r} N_{t}+\log _{2}\left(N_{t} M\right)+2 N_{t}+3 N_{t} \log _{2}(M) \\
& C_{\mathrm{C}}^{\mathrm{IPBSD}}=N_{t} \log _{2}\left(N_{t}\right)+2 N_{t} \log _{2}(M) .
\end{aligned}
$$

\subsection{QBSD algorithm}

By following similar analysis of the PBSD algorithm, the total number of real multiplications, real additions, and comparisons for the QBSD algorithm is given by

$$
\begin{aligned}
C_{\mathrm{M}}^{\mathrm{QBSD}} & =6 N_{r} N_{t}+11 N_{t}+\log _{2}\left(N_{t} M\right) \\
C_{\mathrm{A}}^{\mathrm{QBSD}} & =6 N_{r} N_{t}+\log _{2}\left(N_{t} M\right)+7 N_{t} \\
C_{\mathrm{C}}^{\mathrm{QBS}} & =N_{t} \log _{2}\left(N_{t} M\right) .
\end{aligned}
$$

\subsection{IQBSD}

By following similar analysis of the IPBSD algorithm, the total computational complexity of the IQBSD algorithm can be expressed as

$$
\begin{aligned}
& C_{\mathrm{M}}^{\mathrm{IQBSD}}=6 N_{r} N_{t}+11 N_{t}+\log _{2}\left(N_{t} M\right)+5 N_{t} \log _{2}(M) \\
& C_{\mathrm{A}}^{\mathrm{IQBSD}}=6 N_{r} N_{t}+\log _{2}\left(N_{t} M\right)+7 N_{t}+5 N_{t} \log _{2}(M) \\
& C_{\mathrm{C}}^{\mathrm{IQBSD}}=N_{t} \log _{2}\left(N_{t}\right)+2 N_{t} \log _{2}(M) .
\end{aligned}
$$

In order to make the complexity more intuitional, in Table 1, we have provided the computational complexity of all the proposed algorithms with $N_{t}=16, M=16$, and $N_{r}=8$. Furthermore, by summarizing the above complexity analysis and Table 1 , we have the following two important remarks.

Remark 1. Different from other existing detectors, the length of the candidate list for PBSD and QBSD algorithms does not grow linearly with the modulation order $M$. Thus, it is suitable for coded SM systems with high spectral efficiency, i.e., high modulation order.

Remark 2. Compared with Max-Log-LLR algorithm, the proposed IPBSD and IQBSD algorithms further
Table 1 Computational complexity comparison

\begin{tabular}{llll}
\hline Detectors & Multiplications & Additions & Comparisons \\
\hline Max-Log-LLR & 12,296 & 12,040 & 2048 \\
PBSD & 904 & 808 & 128 \\
IPBSD & 1224 & 1000 & 192 \\
QBSD & 952 & 888 & 128 \\
IQBSD & 1272 & 1208 & 192 \\
\hline
\end{tabular}

reduce the number of multiplications, additions, and comparisons without loss of any performance. In addition, both achieve better performance than the PBSD and QBSD algorithms, respectively, with a neglected complexity overhead.

\section{Simulation results}

In this section, representative numerical simulations are provided to verify the proposed detectors under Rayleigh fading channels to verify the proposed detectors described in the previous sections. Unless otherwise stated, the following set of parameters are used: $N_{t}=4$ and $N_{r}=2$. The code length of LDPC is 360 , and the code rate is $R=\frac{1}{2}$. In addition, the signal to noise ratio is defined as $\mathrm{SNR}=10 \lg \left(\frac{E_{b}}{N_{0}}\right)=10 \lg \left(\frac{E_{s}}{\eta N_{0}}\right)=10 \lg \left(\frac{E_{s}}{N_{0} R \log _{2}\left(N_{t} M\right)}\right)$, where $E_{s}$ is the average energy of the transmit signal.

Figure 4 illustrates the performance difference between the proposed detectors and the Max-Log-LLR detector

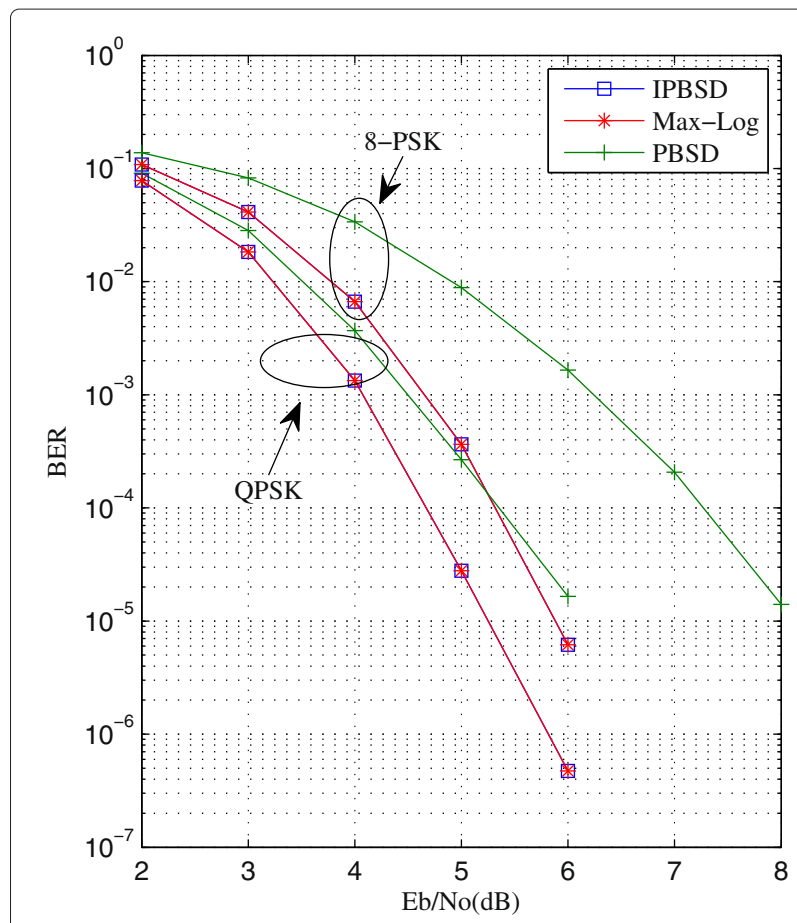

Fig. 4 BER performance comparison between different detectors with PSK modulation 


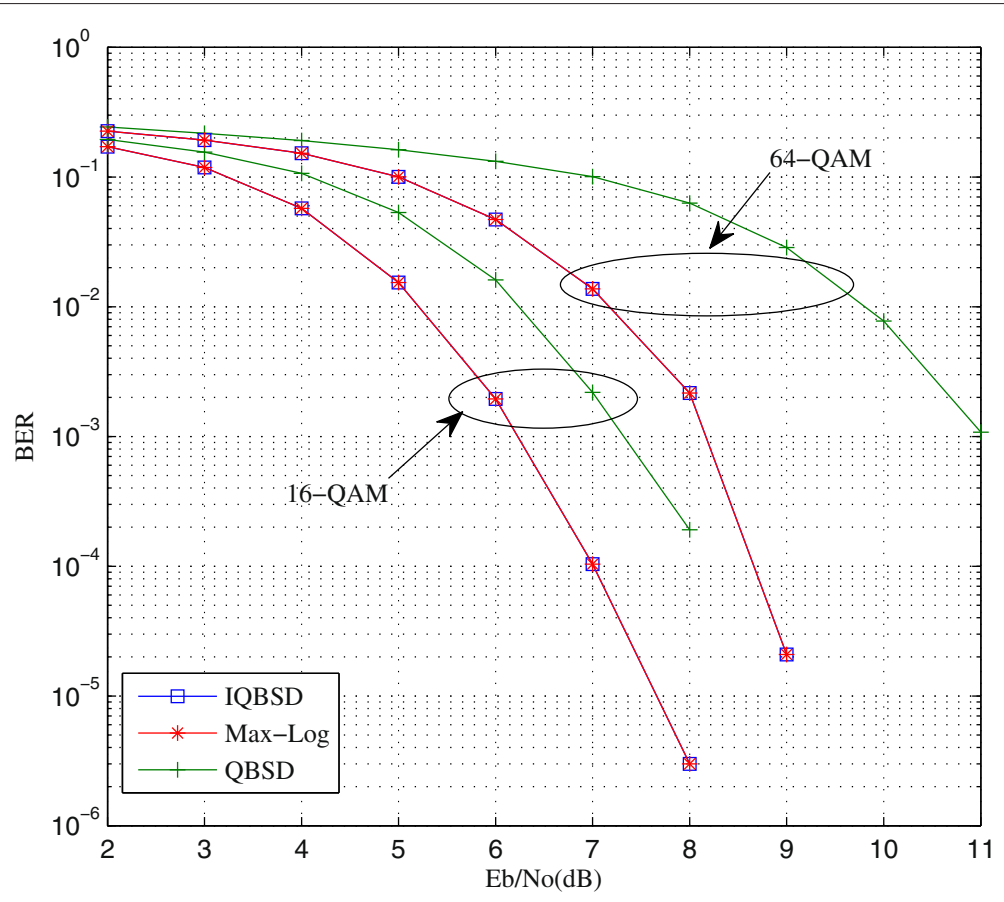

Fig. 5 BER performance comparison between different detectors with QAM modulation

with quadrature phase-shift keying (QPSK) and 8-PSK modulations, respectively. As shown in the figure, compared to Max-Log-LLR algorithm, we find that the proposed PBSD algorithm suffers from about $1.5 \mathrm{~dB}$ performance loss at $10^{-3}$ BER when 8-PSK is employed. While when QPSK is employed, the performance gap is reduced to about $0.4 \mathrm{~dB}$. In addition, we can see that the proposed IPBSD algorithm achieves the same performance as the Max-Log-LLR algorithm with lower computational complexity regardless of the modulation order.

Figure 5 investigates the BER performance of coded SMMIMO systems with 16-QAM and 64-QAM for different detection algorithms. Similar to Fig. 4, it is noted from Fig. 5 that the proposed QBSD algorithm attains much lower computational complexity than Max-Log-LLR algorithm at the expense of performance. For example, at $10^{-3}$ BER, the proposed QBSD algorithm suffers from $1 \mathrm{~dB}$ SNR loss when 16-QAM is employed, and the performance gap becomes large as the modulation order increases. On the other hand, no performance gap is observed between the proposed IQBSD and Max-LogLLR detectors with any modulation order, which validates the effectiveness of our proposed soft-output detection algorithms.

\section{Conclusions}

In this paper, by exploiting the features of $M$-PSK and $M$-QAM constellations, we first have designed two novel low-complexity soft-output algorithms based on the existing hard-decision detection algorithms. The computational complexity of the proposed two detections is much lower than that of Max-Log-LLR algorithm with a acceptable performance degradation. Motivated by this, to further enhance the performance of the two softdecision aided detectors, another two improved algorithms were proposed, i.e., IPBSD and IQBSD detectors. The key idea of the two detectors is finding the counterpart ML estimate for each bit of the modulated symbol. The theoretical analysis and simulation results have shown that the proposed IPBSD and IQBSD detectors achieve exactly the same performance as that of Max-LogLLR detector with reduced computational complexity.

\section{Competing interests}

The authors declare that they have no competing interests.

\section{Acknowledgments}

This work was supported by the National Science Foundation of China under Grant No. 61501507, and the Jiangsu Provincial Natural Science Foundation of China under Grant No. BK20150719.

\section{Received: 29 July 2015 Accepted: 20 December 2015}

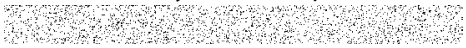

\section{References}

1. R Mesleh, H Haas, S Sinanovic, CW Ahn, S Yun, Spatial modulation. IEEE Trans. Veh. Technol. 57(4), 2228-2241 (2008)

2. M Di Renzo, H Haas, PM Grant, Spatial modulation for multiple antenna wireless system: a survey. IEEE Commun. Mag. 49(12), 182-191 (2011)

3. M Di Renzo, H Haas, A Ghrayeb, S Sugiura, L Hanzo, Spatial modulation for generalized MIMO: challenges, opportunities and implementation. Proc. IEEE. 102(1), 56-103 (2014) 
4. P Yang, M Di Renzo, Y Xiao, S Li, L Hanzo, Design guidelines for spatial modulation. IEEE Commun. Surv. Tutor. 17(1), 6-26 (2014)

5. J Jeganathan, A Ghrayeb, L Szczecinski, Spatial modulation: Optimal detection and performance analysis. IEEE Commun. Lett. 12(8), 545-547 (2008)

6. H Men, M Jin, A low complexity ML detection algorithm for spatial modulation systems with MPSK constellation. IEEE Commun. Lett. 18(8), 1375-1378 (2014)

7. R Rajashekar, KVS Hari, L Hanzo, Reduced-complexity ML detection and capacity-optimized training for spatial modulation systems. IEEE Trans. Commun. 62(1), 112-125 (2014)

8. C Li, Y Huang, M Di Renzo, J wang, Y Cheng, Low-complexity ML detection for spatial modulation MIMO with APSK constellation. IEEE Trans. Veh. Technol. 59(1), 93-104. doi:10.1109/TVT.2015.2430855

9. Q Tang, Y Xiao, P Yang, Q Yu, S Li, A new low complexity near-ML detection algorithm for spatial modulation. IEEE Wirel. Commun. Lett. 2(1), 90-93 (2013)

10. R Mesleh, M Di Renzo, H Haas, P Grant, Trellis coded spatial modulation. IEEE Trans. Wirel. Commun. 9(7), 2349-2361 (2010)

11. E Basar, U Aygolu, E Panayirci, HV Poor, New trellis code design for spatial modulation. IEEE Trans. Wirel. Commun. 10(8), 2670-2680 (2011)

12. M Koca, H Sari, in Proceedings of IEEE PIMRC. Bit-interleaved coded spatial modulation, (Sydney, NSW, 2012), pp. 1949-1954

13. H Liu, J Zheng, J Dou, B Bai, in Proceedings of IEEE ICIST. EXIT-chart-based LDPC code design for spatial modulation, (Shenzhen, China, 2014), pp. 557-560

14. CXu, S Sugiura, SX Ng, L Hanzo, Spatial modulation and space-time shift keying: optimal performance at a reduced detection complexity. IEEE Trans. Commun. 61(1), 206-216 (2013)

15. W Koch, A Baier, in Proceedings of IEEE GLOBECOM. Optimum and sub-optimum detection of coded data disturbed by time-varying intersymbol interference, (San Diego, CA, 1990), pp. 1679-1684

16. Q Wang, Q Xie, Z Wang, S Chen, L Hanzo, A universal low complexity symbol-to-bit soft mapper. IEEE Trans. Veh. Technol. 63(1), 119-130 (2014)

17. NC Beaulieu, A useful integral for wireless communication theory and its application to rectangular signalling constellation error rates. IEEE Trans. Veh. Technol. 54(5), 802-805 (2006)

\section{Submit your manuscript to a SpringerOpen ${ }^{\circ}$ journal and benefit from:}

- Convenient online submission

- Rigorous peer review

- Immediate publication on acceptance

- Open access: articles freely available online

- High visibility within the field

- Retaining the copyright to your article

Submit your next manuscript at springeropen.com 